\title{
Tristetraprolin Inhibits the Growth of Human Glioma Cells through Downregulation of Urokinase Plasminogen Activator/Urokinase Plasminogen Activator Receptor mRNAs
}

\author{
Jinhyun Ryu', Nal Ae Yoon', Yeon Kyung Lee ${ }^{3}$, Joo Yeon Jeong', Seokmin Kang', Hyemin Seong', \\ Jungil Choi ', Nammi Park', Nayoung Kim ${ }^{6}$, Wha Ja Cho', Sun Ha Paek, Gyeong Jae Cho', \\ Wan Sung Choi', Jae-Yong Park, Jeong Woo Park ${ }^{2, *}$, and Sang Soo Kang ${ }^{1, *}$
}

\begin{abstract}
Urokinase plasminogen activator (UPA) and urokinase plasminogen activator receptor (UPAR) play a major role in the infiltrative growth of glioblastoma. Downregulatoion of the UPA and UPAR has been reported to inhibit the growth glioblastoma. Here, we demonstrate that tristetraprolin (TTP) inhibits the growth of U87MG human glioma cells through downregulation of UPA and UPAR. Our results show that expression level of TTP is inversely correlated with those of UPA and UPAR in human glioma cells and tissues. TTP binds to the $A U$-rich elements within the 3' untranslated regions of UPA and UPAR and overexpression of TTP decreased the expression of UPA and UPAR through enhancing the degradation of their mRNAs. In addition, overexpression of TTP inhibited the growth and invasion of U87MG cells. Our findings implicate that TTP can be used as a promising therapeutic target to treat human glioma.
\end{abstract}

\section{INTRODUCTION}

Gliomas are the most common type of primary tumor occurring

${ }^{1}$ Department of Anatomy and Convergence Medical Science, Institute of Health Sciences, School of Medicine, Gyeongsang National University, Jinju 660-751, Korea, '2Department of Biological Sciences, University of Ulsan, Ulsan 680-749, Korea, ${ }^{3}$ Department of Molecular Medicine, Gachon University of Medicine and Science, Incheon 406-840, Korea, ${ }^{4}$ Gyeongnam Department of Environmental Toxicology and Chemistry, Korea Institute of Toxicology (KIT), Jinju 660-844, Korea, ${ }^{5}$ Department of Physiology, Institute of Health Sciences, School of Medicine, Gyeongsang National University, Jinju 660-751, Korea, ${ }^{6}$ Asan Institute for Life Sciences, Asan Medical Center, Seoul 138-736, Korea, ${ }^{7}$ Biomedical Research Center, Ulsan University Hospital, University of Ulsan College of Medicine, Ulsan 682-060, Korea, ${ }^{8}$ Department of Neurosurgery, Seoul National University College of Medicine Seoul 110-744, Korea, ${ }^{9}$ School of Biosystem and Biomedical Science, College of Health Science, Korea University, Seoul 136-703, Korea

*Correspondence: kangss@gnu.ac.kr (SSK); jwpark@ulasan.ac.kr (JWP)

Received 25 September, 2014; revised 28 October, 2014; accepted 30 October, 2014; published online 30 December, 2014

Keywords: glioma, TTP, uPA, uPAR in the central nervous system (Kleihues et al., 1995). In particular, glioblastoma multiforme (GBM), classified by World Health Organization (WHO) as a grade IV glioma, is highly invasive and exhibits rapid growth and a high rate of recurrence (Sehgal, 1998). Because of the malignant nature of GBM, the prognosis and survival rate for patients with unresectable GBM is extremely poor (Gagliano et al., 2005).

The invasiveness of glioma relies on proteolysis of the extracellular matrix during tumor cell invasion. Binding of urokinase plasminogen activator (UPA) to its specific receptor (urokinase plasminogen activator receptor, UPAR) catalyzes the conversion of plasminogen to plasmin, which in turn leads to effective proteolytic effects (Rao, 2003). Overexpression of UPA and UPAR has been observed in the invasive glioblastomas (Yamamoto et al., 2002). Consequently, there have been several efforts to identify agents that reduce UPA and UPAR expression. A previous report showed that resveratrol reduces invasion of U373MG glioma cells through downregulation of UPA and uPAR expression (Ryu et al., 2011). In addition, regulation of UPA and UPAR using small interfering hairpin RNA (siRNA) suppresses glioma cell invasion, angiogenesis, and tumor growth (Gondi et al., 2004).

Stabilization of mRNA that is mediated by AU-rich elements (AREs) located in the 3' untranslated region (3'UTR) appears to play a major role in post-transcriptional regulation of gene expression in tumors (Audic and Hartley, 2004). AREs are distinct sequence elements in the 3'UTR of mRNAs that typically consist of one or more AUUUA pentamers (Barreau et al., 2005). Numerous proteins directly interact with AREs, thereby modulating mRNA stability or translational efficiency (Gruber et al., 2011). The database of ARE-containing mRNAs reveals genes with diverse functions, including proto-oncogenes and growth factors (Bakheet et al., 2001). ARE-binding proteins such as tristetraprolin (TTP or ZFP36) and butyrate response factors 1 (BRF-1) promote degradation of the corresponding transcripts (Lykke-Andersen and Wagner, 2005). Interestingly, TTP expression is suppressed in many cancer cells compared with normal cell types (Brennan et al., 2009).

This study provides evidence for a role of TTP in the suppression of the growth of human glioma cells through downer- 
gulation of UPA and UPAR gene expression. Overexpression of TTP promoted the decay of UPA and UPAR mRNAs through binding to AREs within the 3'UTR of their mRNAs. Furthermore, TTP inhibited growth and invasion of U87MG human glioma cells. These results suggest that UPA and UPAR are physiological targets of TTP in human glioma cells and TTP-mediated downregulation of UPA and UPAR may be used to suppress the progression of glioma.

\section{MATERIALS AND METHODS}

\section{Cells}

U87MG, U373MG, T98G human glioma cells and RAW264.7 mouse macrophage-like cells (ATCC, USA) were maintained in high-glucose Dulbecco's minimal essential medium (DMEM) supplemented with $10 \%$ fetal bovine serum (FBS), $100 \mathrm{U} / \mathrm{ml}$ penicillin, and $100 \mu \mathrm{g} / \mathrm{ml}$ streptomycin at $37^{\circ} \mathrm{C}$ and $5 \% \mathrm{CO}_{2}$.

\section{Human tissue samples}

Human tissue samples were collected during surgical resection in the Department of Neurosurgery, Seoul National University Hospital (SNUH), Korea. All procedures were approved by the Institutional Review Board at SNUH (IRB approval H-OB05036-243) and written consent was obtained from all donors. Samples were snap frozen in liquid nitrogen immediately after resection and stored at $-80^{\circ} \mathrm{C}$ until use.

\section{Adenovirus production and infection}

Adenovirus expressing green fluorescent protein (GFP)-tagged TTP (Ad-TTP-GFP) was prepared and propagated in HEK 293A cells as described previously (Park et al., 2007). After purification by cesium chloride gradient centrifugation, adenoviruses were dialyzed into storage buffer. U87MG cells were transduced with Ad-TTP-GFP (U87MG/Ad-TTP) and incubated at $37^{\circ} \mathrm{C}$ for $48 \mathrm{~h}$ before assays.

\section{Plasmids, transfection, and luciferase assay}

U87MG cells were transfected with TTP-expressing pcDNA6/ V5-HisA vector (pcDNA6/V5-TTP) (Invitrogen, USA) (Lee et al., 2010b) using TurboFect ${ }^{\mathrm{M}}$ in vitro transfection reagent (Fermentas, USA) for overexpression of TTP.

For luciferase assays, U87MG cells were co-transfected with test constructs, psiCHECK-Frag-uPA or UPAR 3'UTR, and pcDNA6/V5-TTP using TurboFect ${ }^{\mathrm{TM}}$ in vitro transfection reagent. Transfected cells were lysed with lysis buffer (Promega, USA) and mixed with luciferase assay reagent (Promega) and the chemiluminescent signal was measured in an Infinite M200 Pro (Tecan, Switzerland). Firefly luciferase signal was normalized to that of Renilla luciferase for each sample. All luciferase assay data reported here represent at least three independent experiments, each consisting of three wells per transfection.

\section{Reverse transcription (RT)-PCR}

Total RNA was extracted using TRIzol reagent (Invitrogen) according to the manufacturer's instructions. Total RNA $(1 \mu \mathrm{g})$ was used for cDNA synthesis in an iCycler thermocycler (BioRad Laboratories, USA). RT-PCR was performed using rTaq polymerase (Elpis biotech, Korea). Primers were designed based on the reported human cDNA sequences for UPA, UPAR, and $h G A P D H$ in the NCBI data bank. Sequences of the primers used for PCR were as follows: TTP: AGGCCAATCGCCACCCCAAA, GTGCCAGGGGCAGCAGAGAA; UPA: ACTTCTCCAACATTCACTGG, ATTCTTCTGGAGGAGAGGAG; UPAR: TGCCCGGGCTCCAATGGTT, ATTCTTCTGGAGGAGAGGAG;
hGAPDH: AGCTGAACGGGAAGCTCACT, TGCTGTAGCCAAATTCGTTG (Bioneer Corporation, Korea).

\section{Quantitative real-time PCR (Q-PCR)}

For RNA kinetic analysis, the amount of UPA and UPAR mRNA was assessed in the presence of actinomycin $D$ by quantitative real-time PCR (Q-PCR) using EvaGreen qPCR Mastermix (Applied Biological Materials Inc., Canada) in a Light Cycler 480 II (Roche Applied Science, USA). The results were evaluated by melting curve analysis and agarose gel electrophoresis. PCR primer pairs were as follows: quPA: CCCTGCCTGCCCTGGAACTC, AGCGGCTITAGGCCCACCTG; quPAR: CATTGACTGCCGAGGCCCCA, TGCTGAAGGCGTCACCCAGG; qh-GAPDH: GCACCCCTGGCCAAGGTCAT, ACGCCACAGTTTCCCGGAGG.

\section{Electrophoretic mobility shift assay (EMSA)}

Biotinylated RNA probes for the wild-type sequence (UPA-AREWT, ACUCCUGUACACUGAAUAGCAUAUUUCACUAUUUUUAUUUAUAUUUUUG-UAAUUUUAAA; UPAR-ARE-WT, GUUGU-UGUUAUUAAUUAAUAUUCAUAUU-AUUUAUUUUAUACUU-ACAUAAAGAUUUUGUACC) were synthesized by Samchully Pharm. Co. Ltd. (Korea). The mutant (MuT) RNA probes as negative control were substituted into AGCA instead of the AUUUA sequences of UPA and UPAR ARE-WT. Cytoplasmic extract was prepared from pcDNA6/V5-TTP-transfected U87-MG cells using NE-PER Nuclear and Cytoplasmic Extraction Reagent (Thermo Pierce Biotechnology Scientific, USA). RNA EMSA was performed using the LightShiftTM Chemiluminescent EMSA Kit.

\section{Western blotting}

Equivalent amounts of total protein (20-30 $\mu \mathrm{g})$ were separated by SDS-PAGE on an $8-12 \%$ polyacrylamide gel and then transferred to a nitrocellulose membrane (Whatman GmbH, Germany) using a semi-dry transfer apparatus (Bio-Rad Laboratories) submerged in transfer buffer (25 mM Tris, $192 \mathrm{mM}$ glycine, $20 \%$ methanol, $\mathrm{pH}$ 8.3). The membrane was blocked with $5 \%$ skim milk in $0.1 \%$ Tween-20/Tris-buffered saline (TTBS) and incubated with the appropriate dilution of primary and secondary antibodies. Antibodies were used: TTP, $\alpha$-tubulin, and $\beta$ actin (Sigma-aldrich, USA), UPA and UPAR (Epitomics, USA). Immunoreactivity was detected by chemiluminescence (ECL; Advansta, USA) using LAS 4000 (Fujifilm, Japan).

\section{Methylthiazoltetrazolium (MTT) assay}

U87MG cells were seeded in 96-well plates at a density of $3 \times$ $10^{3}$ cells/well. After the designated treatment, $100 \mu \mathrm{l}$ of MTT solution $(2 \mathrm{mg} / \mathrm{ml})$ was added to each well, and the cells were incubated at $37^{\circ} \mathrm{C}$ for an additional $2 \mathrm{~h}$. DMSO was added to dissolve the formazan crystals and mixed thoroughly. Absorbance was measured at $570 \mathrm{~nm}$ using an Infinite M200 Pro (Tecan).

\section{Colony formation assay}

For soft agar colony formation assay, cells $\left(5 \times 10^{3}\right)$ were plated in $0.35 \%$ agar medium with $10 \%$ FBS overlaid onto the previously prepared $0.5 \%$ base agar. The medium were changed every 3 days. After 2 weeks, colonies were stained with $0.005 \%$ crystal violet and counted under a light microscope (IX71, Olympus, Japan).

For colony formation assay, cells $\left(1 \times 10^{3}\right)$ were seeded in 6well plate. The medium were changed every 3 days. After 2 weeks, colonies were stained with $0.05 \%$ crystal violet and counted (Ku et al., 2011). 


\section{Cell invasion assay}

Briefly, U87MG cells were seeded on Matrigel-coated Transwell inserts (Corning Incorporated, USA) at a density of $1 \times 10^{5}$ cells/well in triplicate in $100 \mu \mathrm{l}$ DMEM. After 24-h incubation, the filter inserts were removed and the cells were fixed with methanol at $-20^{\circ} \mathrm{C}$ for $5 \mathrm{~min}$ and washed three times with distilled water. Cells were stained with DAPI $(1 \mu \mathrm{g} / \mathrm{ml})$ for $10 \mathrm{~min}$ and rinsed three times with distilled water. The cells on the upper side of the Transwell filter were removed with cotton swabs. The number of invading cells was quantified under a fluorescence microscope (IX71, Olympus).

\section{Statistical analysis}

Data are expressed as mean \pm SEM. Statistical significance was determined using the Student's $t$-test (Prism; GraphPad Software, Inc., USA). $P$ values $<0.05$ were considered statistically significant $\left({ }^{\star} P<0.05,{ }^{* \star} P<0.01\right.$, and $\left.{ }^{\star * \star} P<0.001\right)$.

\section{RESULTS}

TTP overexpression reduces the expression of UPA and UPAR in U87MG human glioma cells

To determine the endogenous level of TTP expression, Western blotting was performed using three human glioma cell lines. TTP protein expression was very low in all three glioma cell lines compared with the positive control [RAW 296.7 mouse macrophage-like cells treated with lipopolysaccharides for $2 \mathrm{~h}$ as recommended by the supplier of the anti-TTP antibody (Sigma-aldrich)] (Fig. 1A). Ad-TTP-GFP virus was transduced into U87MG cells to establish transient expression of TTP-GFP (U87MG/Ad-TTP). As a negative control, U87MG cells were transduced with empty Ad-GFP virus (U87MG/Ad-GFP). Overexpression of TTP in U87MG/Ad-TTP cells was confirmed by RT-PCR and Western blotting (Fig. 1B).

The UPA and UPAR system is known to play a key role in
$\boldsymbol{A}$

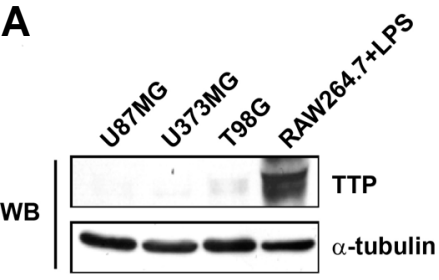

B

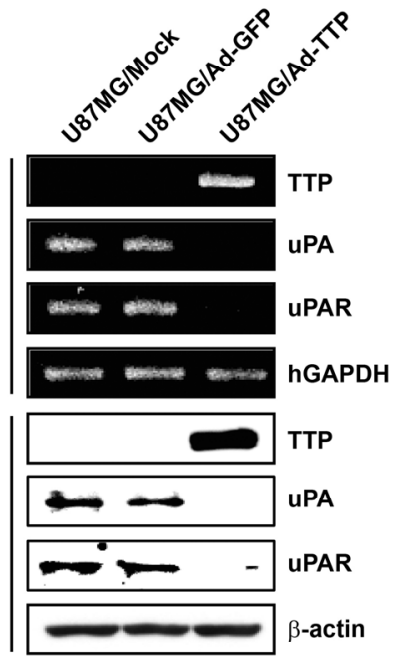

C
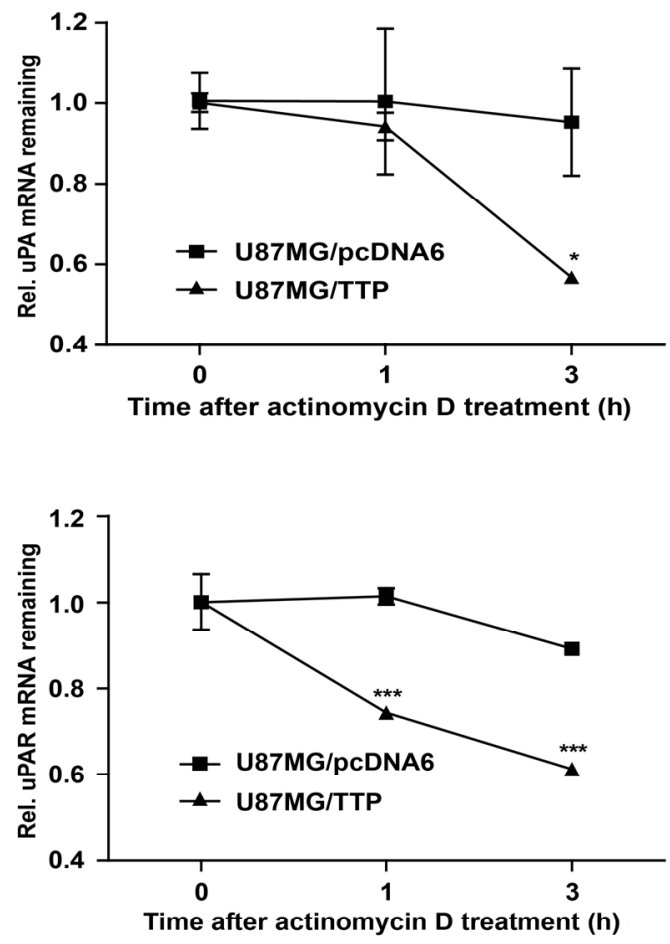

D

WB

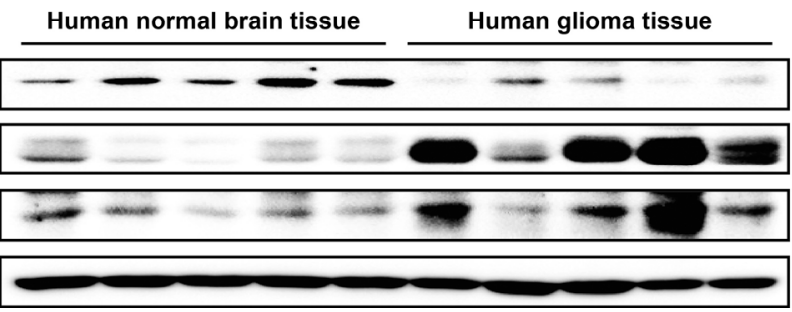

Fig. 1. Overexpression of TTP inhibits UPA and UPAR expression. (A) Endogenous expression of TTP in three human glioma cell lines was determined by Western blotting. RAW264.7 cells that were treated with LPS for $2 \mathrm{~h}$ were used as a positive control. The $\alpha$ tubulin was used as loading control. (B) U87MG cells were transduced with Ad-TTP-GFP or empty Ad-GFP virus. Expression levels of TTP, UPA, and UPAR were determined by RT-PCR and Western blotting. hGAPDH and $\beta$-actin were used as loading controls. (C) U87MG cells were transfected with pcDNA6/N5-TTP vector (U87MG/TTP) or empty pcDNA6N5 vector (U87MG) pcDNA6). The ex-pression of uPA and UPAR mRNAs in U87MG cells was determined by Q-PCR at the indicated times after addition of $5 \mu \mathrm{g} / \mathrm{ml}$ actinomycin D. Data are presented as means \pm SEM. of three independent experiments $\left({ }^{\star} P<0.05\right.$ and ${ }^{\star \star \star} P<$ 0.001). (D) Expression levels of TTP, UPA and UPAR in 9 human normal brain tissues and 10 human glioma tissues were determined by Western blotting. Representative results are shown in this figure. 
cancer progression, invasion, and angiogenesis (Andreasen et al., 2000). In particular, uPA and UPAR are highly expressed in human glioblastoma tissue samples compared with low-grade tumor or normal brain tissue samples (Gladson et al., 1995). In this study, TTP overexpression resulted in decreased expression of both UPA and UPAR in human glioma cells at both mRNA and protein levels (Fig. 1B).

To examine whether reduced expression of UPA and UPAR resulted from decreased stability of UPA and UPAR mRNAs, the half life of these mRNAs was measured by Q-PCR in U87MG cells transfected with pcDNA6/V5-TTP (U87MG/TTP) or pcDNA6/V5 empty vector (U87MG/pcDNA6). After actinomycin $D$ treatment, the half life of UPA mRNA was $1.5 \mathrm{~h}$ in U87MG/TTP cells and $3 \mathrm{~h}$ in U87MG/pcDNA6 cells, respectively. In addition, the half life of UPAR mRNA was $0.5 \mathrm{~h}$ in U87MG/TTP cells and $5.5 \mathrm{~h}$ in U87MG/pcDNA6 cells (Fig. 1C). These results indicate that TTP overexpression significantly induces the decay of UPA and uPAR mRNAs.

TTP expression is inversely correlated with UPA and UPAR expression in human glioma

The expression level of TTP protein was detected by Western blotting in 10 surgically resected human glioma tissues and 9 normal human brain tissues. Representative results are shown in Fig. 1D. In some human glioma tissues from patients, TTP protein expressed low level. However, TTP protein expression level in human glioma tissues was relatively lower than that in normal brain tissues. In contrast to TTP, UPA and UPAR were expressed at higher levels in human glioma tissues than in normal brain tissues (Fig. 1D). These results suggest that TTP expression is inversely correlated with the expression of UPA and UPAR in human glioma.

\section{UPA and UPAR AREs are essential for the inhibitory effect of TTP}

Sequence analysis of the 932-bp 3'UTR of the human uPA transcript reveals the presence of two ARE motifs (Nanbu et al., 1994). The 319-bp sequence corresponding to the 3 'UTR of the human uPAR transcript contains one ARE motif (Roldan et al., 1990). In this study, luciferase reporter constructs containing fragments derived from the 3'UTR of UPA and UPAR mRNAs were cloned downstream of the luciferase reporter gene in the psiCHECK2 luciferase expression vector (Fig. 2A). For TTP overexpression, U87MG cells were transfected with pcDNA6/ V5-TTP (U87MG/TTP). As a negative control, U87MG cells were transfected with an empty pcDNA6/V5 vector (U87MG/ pcDNA6) (Fig. 2B).

To determine whether the 3'UTR of UPA and UPAR mRNAs was required for TTP-mediated destabilization of the respective mRNA, U87MG cells were co-transfected with the psiCHECK2 luciferase expression vector containing fragments of the uPA or UPAR 3'UTR and with pcDNA6/V5-TTP. Luciferase activity of UPA and UPAR was significantly inhibited compared with U87MG cells co-transfected with pcDNA6/V5 empty vector (Fig $2 \mathrm{C})$. These results imply that the 3'UTR of UPA and UPAR mRNAs is involved in the destabilization of UPA and UPAR mRNAs by TTP..
A
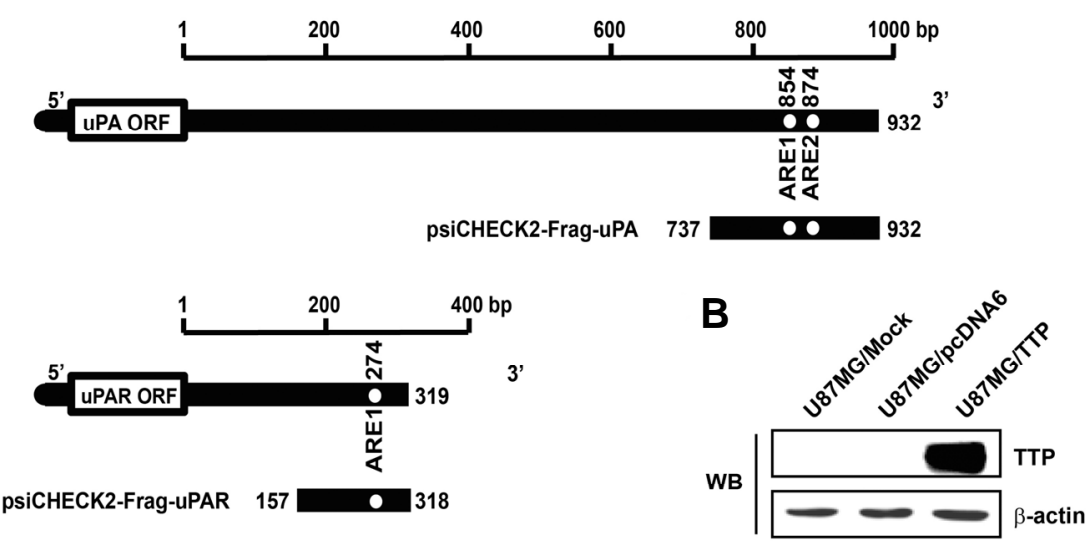

C

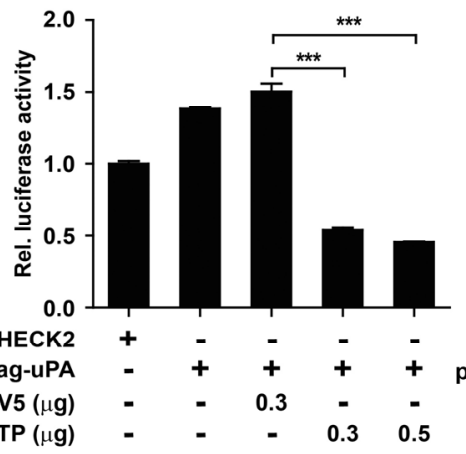

$$
\text { psiCHECK2 }
$$

pcDNA6/V5 $(\mu \mathrm{g})$ pcDNA6/V5-TTP $(\mu g)$
3'

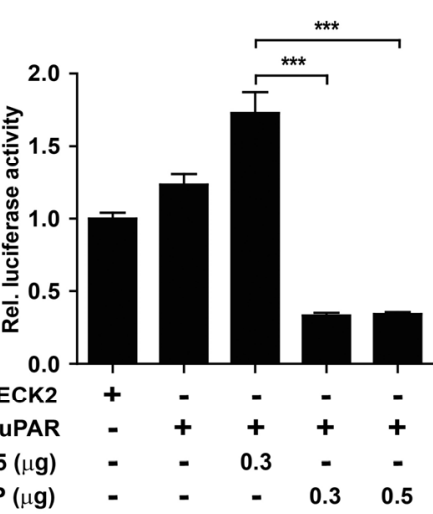

Fig. 2. UPA and UPAR AREs are essential for the inhibitory effect of TTP. (A) Schematic representation of the luciferase reporter constructs used in this study. Fragments derived from the UPA and UPAR mRNA 3'-UTRs were cloned downstream of the luciferase reporter gene in the psiCHECK2 luciferase expression vector. White circles, ARE pentamer motifs (AUUUA). (B) U87MG cells were transfected with pcDNA6/N5-TTP (U87MG/TTP) for overexpression of TTP, or with empty pcDNA6/V5 vector (U87MG/pcDNA6) as a negative control. TTP expression was determined by Westem blotting. The $\beta$-actin was used as loading control. (C) U87MG cells were co-transfected with pcDNA6/N5-TTP or empty vector and $0.3 \mu \mathrm{g}$ psiCHECK2 luciferase reporter construct containing various fragments derived from UPA and UPAR mRNA $3^{\prime} U T R$ as described in (A). Luciferase activity was normalized to firefly luciferase

activity. Results are presented as the mean \pm SEM. of three independent experiments $\left({ }^{\star \star \star} P<0.001\right)$. 


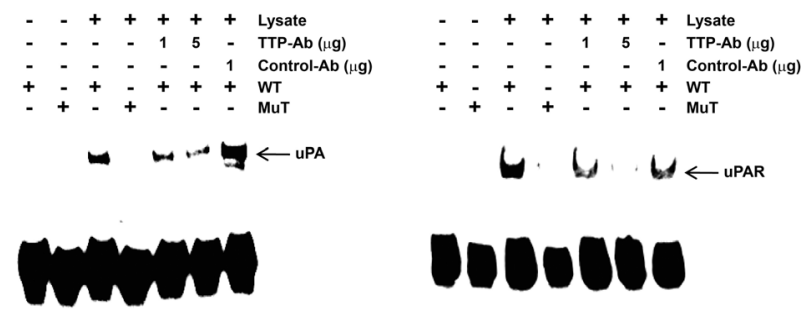

WT : -UAUUUAU-
MUT : -UAGCAU-

Fig. 3. TTP binds to UPA and UPAR AREs. RNA EMSA was performed by mixing cytoplasmic extract containing $1 \mu \mathrm{g}$ of total protein from pcDNA6/N5-TTP-transfected U87MG cells with $20 \mathrm{fmol}$ of biotinylated UPA and UPAR WT (AUUUA) or MuT (AGCA) probes. Anti-TTP or control (anti-mouse lgG) antibody was added to the reaction mixtures as indicated. The binding reactions were separated by electrophoresis on a $5 \%$ polyacrylamide gel under nondenaturing condition. Arrows indicate the position of the RNA-protein complex.

TTP binds to UPA and UPAR AREs

Previous studies showed that TTP binds to the AREs located in the 3'UTR of mRNAs (Cao, 2004). To determine whether TTP directly interacted with AREs within the 3'UTR of UPA and UPAR mRNAs, RNA EMSA was performed using biotinylated RNA probes containing WT or MuT AREs of UPA and UPAR. The RNA probes used in EMSA were the same as those used in the luciferase assay. The MuT RNA probes used as negative controls contained AGCA instead of the AUUUA sequences of UPA and UPAR ARE-WT. Cytoplasmic extract prepared from U87MG cells transfected with pcDNA6/V5-TTP was incubated with biotinylated RNA probes containing WT or MuT AREs of UPA and UPAR $3^{\prime} U T R$. When RNA EMSA was conducted using the UPA-ARE-WT and UPAR-ARE-WT probe, a dominant RNAprotein complex was observed. However, this complex was not formed with UPA-ARE-MuT and UPAR-ARE-MuT. Complex formation was decreased in the presence of anti-TTP antibody (Ab) (Fig. 3). Overall, these results suggest that TTP regulates the expression of UPA and UPAR mRNAs by binding to the ARE in the 3'UTR.

TTP inhibits cell growth and invasion of human glioma cells

Several studies have shown that downregulation of either UPA or uPAR inhibits glioma cell invasion, tumor growth, and angiogenesis (Gondi et al., 2004). To determine whether TTP affects growth and invasion of U87MG cells through decay of UPA and uPAR mRNAs, cell growth and invasion was measured using MTT assay, colony formation assay, and cell inva-
$\boldsymbol{A}$

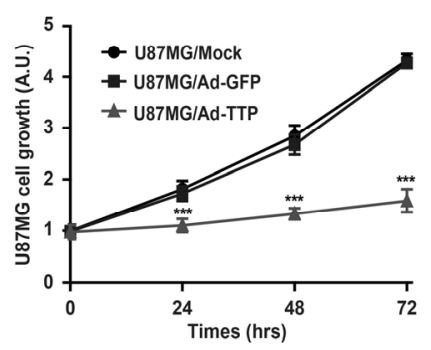

C

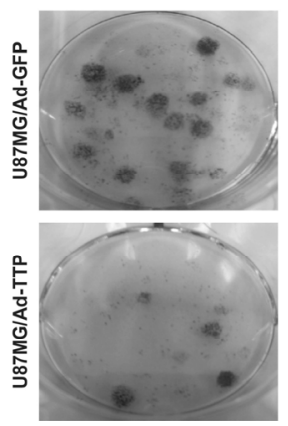

B
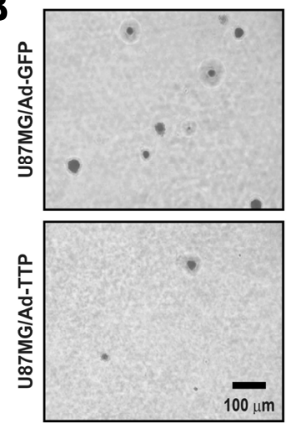

की

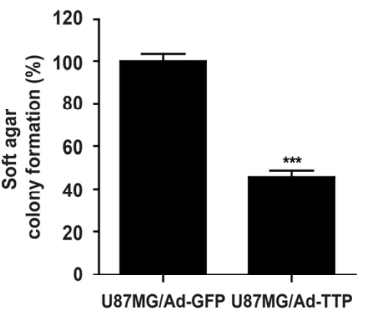

D
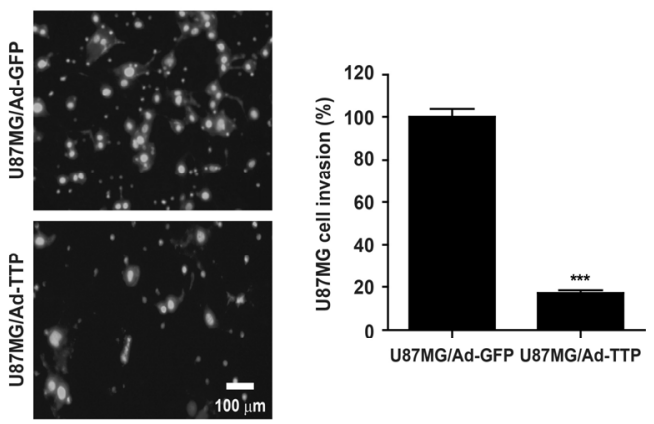

Fig. 4. TTP inhibits growth and invasion of U87MG cells. U87MG cells were transduced with Ad-TTP-GFP (U87MG/Ad-TTP) or empty Ad-GFP (U87MG/Ad-GFP) virus. (A) MTT assay was performed to determine the effect of TTP on cell growth for $72 \mathrm{~h}$. Cell survival relative to that of the control (\%) is presented as the mean \pm SEM. of eight independent experiments $\left.{ }^{* \star \star} P<0.001\right)$. (B) Anchorage-independent growth of U87MG cells. Cells were seeded in soft agar after transduction with Ad-TTP-GFP (U87MG/Ad-TTP) or empty Ad-GFP (U87MG/Ad-GFP) virus. Then, cells were cultured for 2 weeks, colonies were stained with $0.005 \%$ crystal violet. Photographs were taken at a magnification of 100x. Colonies were counted and represented as the mean \pm SEM. of three independent experiments $\left({ }^{* \star *} P<0.001\right)$. (C) Anchorage-dependent growth of U87MG cells. Cells were seeded in 6 -well plate and cultured for 2 weeks. After 2 weeks, colonies were stained with $0.05 \%$ crystal violet. Colonies were counted and represented as the mean \pm SEM. of three independent experiments $\left({ }^{*} P<0.05\right)$. (D) Cell invasion was assessed by matrigel invasion assay. Data are presented as the mean \pm SEM. of three independent experiments $\left({ }^{\star \star \star} P<0.001\right)$. 
sion assay in U87MG cells transduced with Ad-TTP-GFP (U87MG/Ad-TTP) or empty Ad-GFP virus (U87MG/Ad-GFP). U87MG/Ad-TTP cell growth determined by MTT assay for $72 \mathrm{~h}$ was suppressed in U87MG/Ad-TTP cells (Fig. 4A). Overexpression of TTP also inhibited colony-forming ability of human glioma cells. The size and numbers of colonies in anchorageindependent (Fig. 4B) and -dependent manners (Fig. 4C) were decreased in U87MG/Ad-TTP cells. Moreover, cell invasion was suppressed in U87MG/Ad-TTP cells compared with U87MG/Ad-GFP cells (Fig. 4D). These results show that TTP inhibits cell growth and invasion in human glioma by increasing the decay of UPA and UPAR mRNAs.

\section{DISCUSSION}

TTP, a tandem $\mathrm{CCCH}$ zinc-finger RNA-binding protein, regulates the stability of several ARE-containing mRNAs (Baou et al., 2009). Previous studies reported that TTP expression is suppressed in many cancer cells compared with normal cell types. Moreover, a lack of TTP is associated with a variety of cancer-related processes (Brennan et al., 2009). In this respect, regulation of TTP expression has been shown to play a role in several cancers such as colon, breast, skin, lung, and brain (Lee et al., 2013; Suswam et al., 2008). However, its role in glioma has not been fully investigated.

In the present study, we demonstrated very low endogenous expression of TTP in three human glioma cell lines, U87MG, U373MG, and T98G (Fig. 1A). Furthermore, TTP protein expression in human glioma tissues was relatively lower than that in normal brain tissues (Fig. 1D). Previous studies have identified underlying mechanisms linking a deficiency of TTP with cancer. A number of micro RNAs that are overexpressed in the tissue of breast cancer patients, such as miR-29a, bind specifically to the 3'UTR of TTP mRNA and reduce its expression (AlAhmadi et al., 2013). In addition, phosphorylation of TTP by the p38 MAPK/MK2 pathway induces removal of TTP protein by proteasomes (Brook et al., 2006) and leads to the progression of tumors through increased mRNA stability of VEGF and IL-8, which are normally downregulated by TTP (Suswam et al., 2008).

The production of pro-uPA and its activation to UPA by binding to UPAR plays a key role in glioma cell invasion because UPA is required for effective activation of plasmin to plasminogen and subsequent degradation of the extracellular matrix (Ellis and Dano, 1993). The expression of both UPA and UPAR correlates with an invasive cancer cell phenotype and poor prognosis. UPA and UPAR are highly expressed in human glioblastoma tissue samples compared with low-grade or normal brain tissue samples (Gladson et al., 1995). Moreover, TTP has been shown to regulate uPA and UPAR expression in invasive breast cancer cells (Al-Souhibani et al., 2010). In the present study, transient overexpression of TTP in U87MG glioma cells was achieved using Ad-GFP virus (Fig. 1B) or pcDNA6/V5 vector (Fig. 2B). Our data demonstrated that overexpression of TTP reduced UPA and UPAR expression (Fig. 1B) and destabilized their mRNAs (Fig. 1C). In addition, in contrast to the low level of endogenous expression of TTP, UPA and UPAR were expressed at higher levels in human glioma tissues compared with normal brain tissues (Fig. 1D), indicating that TTP expression is inversely correlated with UPA and UPAR expression in human glioma tissues.

TTP is an ARE-binding protein that induces decay of AREcontaining mRNAs (Lee et al., 2010a). Genes that contain AREs in their 3'UTR include growth factors and protooncogenes (c-fos, c-myc, and VEGF) (Bakheet et al., 2001) as well as invasion-associated genes (UPA, UPAR, and MMP-11) (Al-Souhibani et al., 2010). Recent studies have been shown TTP controls the decay of growth factors and proto-oncogenes (Marderosian et al., 2006). The present study demonstrates that TTP negatively regulates the expression of UPA and UPAR through direct binding to the AREs within the $3^{\prime} \mathrm{UTR}$ of their mRNAs. Specifically, the expression of luciferase reporter genes containing the UPA or UPAR 3'UTR was inhibited by TTP overexpression (Fig. $2 \mathrm{C}$ ) and RNA EMSA showed that TTP interacted with ARE regions within the 3'UTR of UPA and UPAR mRNAs (Fig. 3).

Several anticancer therapeutic approaches targeting uPA and UPAR function using selective inhibitors of UPA activity, antagonist peptides, monoclonal antibodies that suppress UPA binding to UPAR, and gene therapy techniques silencing uPA and UPAR expression have been shown to have antitumor effects in xenograft models (Ulisse et al., 2009). More recently, regulation of UPA and UPAR using antisense or small hairpin RNA (shRNA) was shown to inhibit cell invasion, tumor growth, and angiogenesis in human glioma cells (Gondi et al., 2004). In the current study, TTP overexpression inhibited growth (Figs. 4A4C) and invasion (Fig. 4D) of U87MG cells. Consistent with these findings, expression of TTP has been shown to suppress cell growth and invasion in brain and colon cancer cells (Selmi et al., 2012; Stoecklin et al., 2003). These findings indicate that TTP performs a tumor suppressor function by regulating the mRNA stability of ARE-containing genes such as UPA and UPAR.

In summary, this study demonstrates that TTP expression was inversely correlated with UPA and UPAR expression in human glioma cells and tissues. Moreover, TTP destabilized UPA and UPAR mRNAs by binding to the ARE regions within the $3^{\prime} \mathrm{UTR}$ of their mRNAs. Furthermore, upregulation of TTP expression suppressed growth and invasion of U87MG cells. These findings implicate regulation of TTP expression as a promising therapeutic tool for human glioma, and further studies of the role in TTP in human glioma are therefore warranted.

\section{ACKNOWLEDGMENTS}

This work was supported by the National Research Foundation of Korea (NRF) and by a grant funded by the Korean Government [Ministry of Education and Science Technology (MEST)] (No. 2013R1A2A2A01068964).

\section{REFERENCES}

Al-Ahmadi, W., Al-Ghamdi, M., Al-Souhibani, N., and Khabar, K.S. (2013). miR-29a inhibition normalizes HuR over-expression and aberrant AU-rich mRNA stability in invasive cancer. J. Pathol. 230, 28-38.

Al-Souhibani, N., Al-Ahmadi, W., Hesketh, J.E., Blackshear, P.J., and Khabar, K.S. (2010). The RNA-binding zinc-finger protein tristetraprolin regulates AU-rich mRNAs involved in breast cancer-related processes. Oncogene 29, 4205-4215.

Andreasen, P.A., Egelund, R., and Petersen, H.H. (2000). The plasminogen activation system in tumor growth, invasion, and metastasis. Cell Mol. Life Sci. 57, 25-40.

Audic, Y., and Hartley, R. (2004). Post-transcriptional regulation in cancer. Biol. Cell 96, 479-498.

Bakheet, T., Frevel, M., Williams, B.R., Greer, W., and Khabar, K.S. (2001). ARED: human AU-rich element-containing mRNA database reveals an unexpectedly diverse functional repertoire of encoded proteins. Nucleic Acids Res. 29, 246-254.

Baou, M., Jewell, A., and Murphy, J.J. (2009). TIS11 family proteins and their roles in posttranscriptional gene regulation. J. Biomed. Biotechnol. 2009, 634520.

Barreau, C., Paillard, L., and Osborme, H.B. (2005). AU-rich elements 
and associated factors: are there unifying principles? Nucleic Acids Res. 33, 7138-7150.

Brennan, S.E., Kuwano, Y., Alkharouf, N., Blackshear, P.J., Gorospe, M., and Wilson, G.M. (2009). The mRNA-destabilizing protein tristetraprolin is suppressed in many cancers, altering tumorigenic phenotypes and patient prognosis. Cancer Res. 69, 5168-5176.

Brook, M., Tchen, C.R., Santalucia, T., Mcllrath, J., Arthur, J.S., Saklatvala, J., and Clark, A.R. (2006). Posttranslational regulation of triste-traprolin subcellular localization and protein stability by p38 mitogen-activated protein kinase and extracellular signalregulated kinase pathways. Mol. Cell. Biol. 26, 2408-2418.

Cao, H. (2004). Expression, purification, and biochemical characterization of the antiinflammatory tristetraprolin: a zinc-dependent mRNA binding protein affected by posttranslational modifications. Biochemistry 43, 13724-13738.

Ellis, V., and Dano, K. (1993). Potentiation of plasminogen activation by an anti-urokinase monoclonal antibody due to ternary complex formation. A mechanistic model for receptor-mediated plasminogen activation. J. Biol. Chem. 268, 4806-4813.

Gagliano, N., Moscheni, C., Torri, C., Magnani, I., Bertelli, A.A., and Gioia, M. (2005). Effect of resveratrol on matrix metalloproteinase-2 (MMP-2) and secreted protein acidic and rich in cysteine (SPARC) on human cultured glioblastoma cells. Biomed. Pharmacother. 59, 359-364.

Gladson, C.L., Pijuan-Thompson, V., Olman, M.A., Gillespie, G.Y., and Yacoub, I.Z. (1995). Up-regulation of urokinase and urokinase receptor genes in malignant astrocytoma. Am. J. Pathol. 146, $1150-1160$.

Gondi, C.S., Lakka, S.S., Dinh, D.H., Olivero, W.C., Gujrati, M., and Rao, J.S. (2004). Downregulation of UPA, UPAR and MMP-9 using small, interfering, hairpin RNA (siRNA) inhibits glioma cell invasion, angiogenesis and tumor growth. Neuron Glia Biol. 1, 165-176.

Gruber, A.R., Fallmann, J., Kratochvill, F., Kovarik, P., and Hofacker, I.L. (2011). AREsite: a database for the comprehensive investigation of AU-rich elements. Nucleic Acids Res. 39, D66-69.

Kleihues, P., Soylemezoglu, F., Schauble, B., Scheithauer, B.W., and Burger, P.C. (1995). Histopathology, classification, and grading of gliomas. Glia 15, 211-221.

Ku, B.M., Lee, Y.K., Jeong, J.Y., Ryu, J., Choi, J., Kim, J.S., Cho, Y.W., Roh, G.S., Kim, H.J., Cho, G.J., et al. (2011). Caffeine inhibits cell proliferation and regulates PKA/GSK3beta pathways in U87MG human glioma cells. Mol. Cells 31, 275-279.

Lee, H.H., Son, Y.J., Lee, W.H., Park, Y.W., Chae, S.W., Cho, W.J., Kim, Y.M., Choi, H.J., Choi, D.H., Jung, S.W., et al. (2010a) Tristetraprolin regulates expression of VEGF and tumorigenesis in human colon cancer. Int. J. Cancer 126, 1817-1827.

Lee, H.H., Vo, M.T., Kim, H.J., Lee, U.H., Kim, C.W., Kim, H.K., Ko, M.S., Lee, W.H., Cha, S.J., Min, Y.J., et al. (2010b). Stability of the LATS2 tumor suppressor gene is regulated by tristetraprolin. J. Biol. Chem. 285, 17329-17337.

Lee, H.H., Yang, S.S., Vo, M.T., Cho, W.J., Lee, B.J., Leem, S.H.,
Lee, S.H., Cha, H.J., and Park, J.W. (2013). Tristetraprolin down-regulates IL-23 expression in colon cancer cells. Mol. Cells 36, 571-576.

Lykke-Andersen, J., and Wagner, E. (2005). Recruitment and activation of mRNA decay enzymes by two ARE-mediated decay activation domains in the proteins TTP and BRF-1. Genes Dev. 19, 351-361.

Marderosian, M., Sharma, A., Funk, A.P., Vartanian, R., Masri, J., Jo, O.D., and Gera, J.F. (2006). Tristetraprolin regulates cyclin D1 and c-Myc mRNA stability in response to rapamycin in an Akt-dependent manner via p38 MAPK signaling. Oncogene 25 6277-6290.

Nanbu, R., Menoud, P.A., and Nagamine, Y. (1994). Multiple instability-regulating sites in the $3^{\prime}$ untranslated region of the urokinase-type plasminogen activator mRNA. Mol. Cell. Biol. 14, 4920-4928.

Park, J.Y., Hwang, E.M., Park, N., Kim, E., Kim, D.G., Kang, D., Han, J., Choi, W.S., Ryu, P.D., and Hong, S.G. (2007). Gateway RFP-fusion vectors for high throughput functional analysis of genes. Mol. Cells 23, 357-362.

Rao, J.S. (2003). Molecular mechanisms of glioma invasiveness: the role of proteases. Nat. Rev. Cancer 3, 489-501.

Roldan, A.L., Cubellis, M.V., Masucci, M.T., Behrendt, N., Lund, L.R., Dano, K., Appella, E., and Blasi, F. (1990). Cloning and expression of the receptor for human urokinase plasminogen activator, a central molecule in cell surface, plasmin dependent proteolysis. EMBO J. 9, 467-474.

Ryu, J., Ku, B.M., Lee, Y.K., Jeong, J.Y., Kang, S., Choi, J., Yang Y., Lee, D.H., Roh, G.S., Kim, H.J., et al. (2011). Resveratro reduces TNF-alpha-induced U373MG human glioma cell invasion through regulating NF-kappaB activation and UPA/UPAR expression. Anticancer Res. 31, 4223-4230.

Sehgal, A. (1998). Molecular changes during the genesis of human gliomas. Semin. Surg. Oncol. 14, 3-12.

Selmi, T., Martello, A., Vignudelli, T., Ferrari, E., Grande, A Gemelli, C., Salomoni, P., Ferrari, S., and Zanocco-Marani, T. (2012). ZFP36 expression impairs glioblastoma cell lines viability and invasiveness by targeting multiple signal transduction pathways. Cell Cycle 11, 1977-1987.

Stoecklin, G., Gross, B., Ming, X.F., and Moroni, C. (2003). A novel mechanism of tumor suppression by destabilizing AU-rich growth factor mRNA. Oncogene 22, 3554-3561.

Suswam, E., Li, Y., Zhang, X., Gillespie, G.Y., Li, X., Shacka, J.J., Lu, L., Zheng, L., and King, P.H. (2008). Tristetraprolin downregulates interleukin-8 and vascular endothelial growth factor in malignant glioma cells. Cancer Res. 68, 674-682.

Ulisse, S., Baldini, E., Sorrenti, S., and D'Armiento, M. (2009). The urokinase plasminogen activator system: a target for anti-cancer therapy. Curr. Cancer Drug Targets 9, 32-71.

Yamamoto, M., Ueno, Y., Hayashi, S., and Fukushima, T. (2002). The role of proteolysis in tumor invasiveness in glioblastoma and metastatic brain tumors. Anticancer Res. 22, 4265-4268. 